\title{
OSAS due to massive lingual tonsillar hypertrophy in 8-year-old boy with delta-beta-thalassemia - a case report
}

\begin{abstract}
A few case reports describe an association of airway obstruction due to lingual tonsillar hypertrophy in patients with thalassemia. We present an eight-year-old boy with a history of delta-beta-thalassemia and recurring lingual tonsillar hypertrophy. We hypothesize that this could be the result of extramedullary hematopoiesis.
\end{abstract}

Keywords: obstructive sleep apnea syndrome, beta-delta-thalassemia, lingual tonsillar hypertrophy, extramedullary hematopoiesis
Volume 2 Issue 4 - 2017

\author{
Willems RPP,' Van Waarden burg DA, ${ }^{2}$ \\ Bergshoeff $E,{ }^{3}$ Busari OJ \\ IDepartment of Pediatrics, Maastricht University Medical \\ Center, Netherlands \\ ${ }^{2}$ Department of E.N.T., Atrium Medical Center, Netherlands \\ ${ }^{3}$ Department of Pediatrics, Zuyderland Hospital, Netherlands
}

Correspondence: Willems RPP, Maastricht University Medical
Center+, P. Debyelaan 25, 6229 HX Maastricht, Netherlands, Email rpp.willems@alumni.maastrichtuniversity.nl

Received: December 17, 2014 | Published: July 18, 2015
Abbreviations: OSAS, obstructive sleep apnea syndrome; E.N.T, ear, nose, throat; AHI, apnea-hypopnea index; ODI, oxygendesaturation index; CPAP, continuous positive airway pressure

\section{Introduction}

We present a case of an obstructive sleep apnea syndrome (OSAS) due to extensive lingual tonsillar hypertrophy in an eight-year-old boy with a 2-year history of a heterozygous delta-beta-thalassemia for which he used folic acid (0,5 mg once daily) (Figure 1$)$.

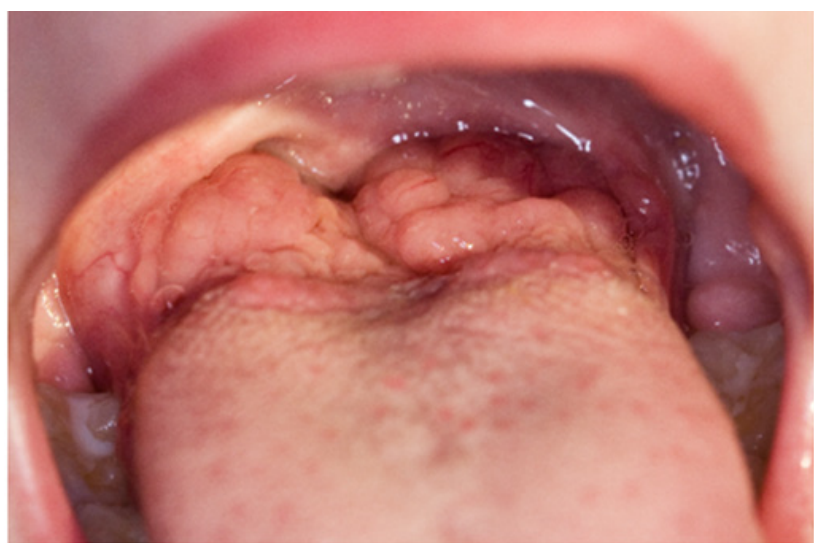

Figure I Massive lingual tonsillar hypertrophy in an eight-year-old boy with delta-beta-thalassemia.

\section{Case presentation}

Prior to the diagnosis of the delta-beta-thalassemia in 2006, the boy had been seeing the E.N.T.-surgeon because of nocturnal snoring, hypernasal speech, difficulties with swallowing and open-mouth breathing. In 2007, he underwent an adenoidectomy but presented a year later, again with similar complaints and with glue-ears. An adenotonsillectomy was performed and tympanostomy tubes were placed. At the time of surgery there was marked hypertrophy of tonsils (kissing tonsils). In October 2009 there was a relapse of the snoring complaints for which a third surgery (adenoidectomy) was performed in 2010 .
In 2012, the boy presented to the E.N.T. surgeon due to a relapse of progressive snoring complaints. At this time his sleep was seriously disturbed. His parents described an obstructive breathing and episodes of apneas during sleep, without cyanosis. During the day he was often very tired and had concentration difficulties at school. He had also gained weight despite swallowing difficulties but without complaints of dysphagia. On inspection of the oropharynx, the E.N.T.-surgeon discovered severe hypertrophy of the lingual tonsils that extended to the soft palate and obstructed almost the entire posterior oropharynx. There was no cervical lymfadenopathy. RAST-screening for allergies was negative.

As this was a fourth episode of lymphoid hyperplasia in the child's oropharynx, it was decided to perform additional investigations to determine the etiology of the problem before performing any further surgical intervention. The differential diagnosis at this stage was that of a (chronic) infection, gastroesopheal reflux, malignancy (lymphoma) or immune deficiency.

The boy was referred to a tertiary center for an overnight polysomnography that revealed an apnea-hypopnea index (AHI) of 4.0 (6.5 when lying on his back), and an oxygen-desaturation index (ODI) of 0.4. The diagnosis of a mild obstructive sleep apnea syndrome (OSAS) was made. MRI scans of the head and neck region were conducted and showed significant swelling of patient's lingual tonsils.

Due to the severity of his symptoms, a laser-assisted partial surgical resection of the hypertrophic lingual tonsils was performed. Histopathological examination of tissue biopsies revealed no evidence of malignancy but only lymphoid hyperplasia. Throat swab cultures showed Streptococcus Pyogenes. Culture results for BartonellaHenselae and Actinomyces were negative. Our patient did receive amoxicilline for a duration of 6 weeks. Because of a suspicion of gastro-oesophageal reflux disease anti-reflux therapy was prescribed (omeprazol and domperidone) postoperatively.

Unfortunately within a year a relapse of the signs and symptoms of upper airway obstruction occurred. A polysomnography this time was supportive of severe obstructive sleep apnea syndrome (AHI 14; 
ODI 18) and continuous positive airway pressure (CPAP) during sleep was started. An MRI showed enlargement of the lymphoid tissue of the oro- and nasopharynx and neck region. Again there was massive lingual tonsillar hypertrophy. Our differential diagnosis at this stage was reviewed and included immunological disorders, malignancy or extramedullary hematopoiesis. Further investigations are needed to determine the cause of our patient's disorder.

\section{Discussion}

We describe a child with delta-beta-thalassemia with obstructive sleep apnea syndrome (OSAS) due to lingual tonsillar hypertrophy. Consisting of lymphoid tissue, the lingual tonsils are located at the base of the tongue between the circumvallate papilla anteriorly and the epiglottis posteriorly. When enlarged, the lingual tonsils appear as 2 masses divided by the glossoepiglottic fold. Varying in size, they may become so distinct that they displace the epiglottis posteriorly. Patients with lingual tonsillar hypertrophy may present with a number of symptoms including sore throat, dysphagia, globus sensation, snoring, alteration of voice, chronic cough, snoring, and obstructive sleep apnea. ${ }^{1}$ Although adenotonsillar hypertrophy has been reported to be the most common cause of pediatric obstructive sleep apnea syndrome (OSAS), enlargement of the lingual tonsils is increasingly being recognized as a cause of OSAS, even after adenotonsillectomy. ${ }^{1-3}$ Up to two thirds of patients with lingual tonsillar hypertrophy have undergone a palatal tonsillectomy or adenoidectomy. Lingual tonsillar hypertrophy is also more often encountered in obese children, ${ }^{1,3}$ patients with Down syndrome ${ }^{2,4}$ and mucopolysaccharidoses, ${ }^{2}$ and is noted to be one of the reasons for unexpected difficult airway intubation in children. ${ }^{1}$

Obstructive sleep apnea syndrome (OSAS) in children is a disorder characterized by prolonged partial upper airway obstruction or intermittent complete obstruction that disrupts normal breathing during sleep and the normal sleeping pattern. OSAS is associated with snoring, chronic mouth breathing, and restlessness during sleep with or without frequent awakening. ${ }^{5}$ The sleep disruption may result in excessive daytime sleepiness, ${ }^{6}$ behavioral problems and learning disorders. However OSAS may also have more serious complications like growth retardation, neurological and cardiovascular disorders. The gold standard diagnostic test in OSAS is polysomnography and the most widely used objective measure of the severity of OSAS is the apnea-hypopnea index (AHI). OSAS in children is categorized as absent (AHI, <1), mild (AHI, 1-5), moderate (AHI, 5-10), or severe (AHI, >10). ${ }^{7}$ After adenotonsillectomy a post-operative airway obstruction may occur due to acute edema of the tongue base. ${ }^{2}$ Although the treatment of first choice in children with OSAS consists of adenotonsillectomy, obese patients often show persistent, at least mild, postoperative OSAS in $75 \%$ of cases. ${ }^{7}$

Beta-thalassemia is a hereditary hematological disease characterized, in the case of major and intermediate types, by severe and chronic hemolytic anemia and ineffective erythropoiesis. Betathalassemia is a genetic disorder resulting in inadequate or complete absent beta-globin production, and an imbalance in alpha- and betaglobin chain production. Patient with beta-thalassemia often have bone deformities, growth abnormalities and hepatosplenomegaly. Ineffective erythropoiesis causes osteoporosis and extreme expansion of marrow space in the skull, long bones, and facial bones. ${ }^{6,8}$ It is most often seen in Southeast Asia but also other parts of the world. ${ }^{8}$ However, our patient is known with delta-beta-thalassemia. Deltabeta-thalassemia is a form of beta-thalassemia characterized by decreased or absent synthesis of the delta- and beta-globin chains with a compensatory increase in expression of fetal gamma-chain synthesis. One can have a disease close to the clinical picture of beta-thalassaemia intermedia. The condition is found in many ethnic groups but is most common in Greece and Italy.

There are increasing reports in the literature suggesting that extramedullary hematopoeisis may also be a etiological cause of oropharyngeal lymphoid hyperplasia. However most of the cases reported so far have exclusively been in children. Kapelushnik et. al. published a case report of a child with thalassemia intermedia in which extramedullary hematopoiesis resulted in obstruction of the nasopharynx and caused OSAS. Blood transfusions were used to treat this symptomatic extramedullary hematopoiesis. Other proposed treatment modalities included radiation therapy and hydroxyurea. ${ }^{5} \mathrm{~A}$ study of Sritippayawan et al., ${ }^{8}$ showed that the estimated prevalence of OSAS in children with severe beta-thalassemia was high (8.3\%) (higher than reported in children without thalassemia). Reactive lymphoid hyperplasia was demonstrated in all cases. In this study a high serum ferritin level was associated with the presence of OSAS. An increased serum ferritin level is generally found in patients with severe betathalassemia who require frequent blood transfusions. They suggest that children with severe beta-thalassemia and snoring or OSAS symptoms should be periodically assessed by polysomnography. ${ }^{8}$ Tarasiuk et al., ${ }^{6}$ performed nocturnal polysomnographic studies in ten children and adolescents with beta-thalassemia and found a 2 -fold increase of arousals and awakenings in patients with chronic anemia compared with healthy volunteers. There is evidence that treatment of iron deficiency anemia improves the quality of life and subjective as well as objective sleep quality. However, there is no evidence for a reduction of periods of apnea and hypopnea by treatment of iron deficiency anemia. ${ }^{6}$ While most of the cases reported have linked the discovered extramedullary hyperplasia to thalassemia, there has been none yet that has reported this association with delta-beta-thalassemia.

\section{Summary}

In summary, the patient presented in this case was known with a delta-beta-thalassemia. At presentation, his serum hemoglobin level was $7.5 \mathrm{mmol} / \mathrm{L}$ and the $\mathrm{MCV}$ was $60 \mathrm{fl}$. Repeated serum ferrintin levels were 15 and $23 \mu \mathrm{g} / \mathrm{L}$ and iron levels 7.8 and 12.5 $\mathrm{mmol} / \mathrm{L}$ respectively. After iron suppletion, his serum ferritin levels normalized. There were also complaints of severe recurrent OSAS due to the lymphoid hyperplasia of the lingual tonsils. Despite extensive investigation however, we could only hypothesize a possible association with an extramedullary hematopoiesis based on the case reports found in the literature.

\section{Conclusion}

In conclusion, children with delta-beta-thalassemia may present with an extensive lymphoid hyperplasia of Waldeyer's ring which may lead to severe OSAS. The development of hyperplastic tissue may be induced by extramedullary hematopoiesis, in some cases reflected by a high serum ferritin level. Surgical treatment options include adenotonsillectomy and laser-assisted resection of the hyperplastic lingual tonsils. Further investigation is needed to elucidate the mechanisms behind this pathophysiological process.

\section{Acknowledgments}

None.

\section{Conflicts of interest}

None. 


\section{Funding}

None.

\section{References}

1. Patel AB, Davidian E, Reebye U. Complicated airway due to unexpected lingual tonsil hypertrophy. Anesth Prog. 2012;59(2):82-84.

2. Abdel-Aziz M, Ibrahim N, Ahmed A, et al.) Lingual tonsils hypertrophy; a cause of obstructive sleep apnea in children after adenotonsillectomy: operative problems and management. Int $J$ Pediatr Otorhinolaryngol. 2011;75(9):1127-1131.

3. Guimaraes CV, Kalra M, Donnelly LF, et al. The frequency of lingual tonsil enlargement in obese children. Am $J$ Roentgenol. 2008;190(4):973-975.

4. Sedaghat AR, Flax-Goldenberg RB, Gayler BW, et al. A case-control comparison of lingual tonsillar size in children with and without Down syndrome. Laryngoscope. 2012;122(5):1165-1169.
5. Kapelushnik J, Shalev H, Schulman H, et al. Upper airway obstructionrelated sleep apnea in a child with thalassemia intermedia. $J$ Pediatr Hematol Oncol. 2001;23(8):525-526.

6. Tarasiuk A, Abdul-Hai A, Moser A, et al. Sleep disruption and objective sleepiness in children with beta-thalassemia and congenital dyserythropoietic anemia. Arch Pediatr Adolesc Med. 2003; 157(5):463-468.

7. Chan DK, Jan TA, and Koltai PJ. Effect of obesity and medical comorbidities on outcomes after adjunct surgery for obstructive sleep apnea in cases of adenotonsillectomy failure. Arch Otolaryngol Head Neck Surg. 2012;138(10):891-896.

8. Sritippayawan S, Norasetthekul S, Nuchprayoon I, et al. Obstructive sleep apnea among children with severe beta-thalassemia. Southeast Asian J Trop Med Public Health. 2012;43(1):152-159. 